Davendra P.S. Sohal and Alok A. Khorana, Cleveland Clinic, Cleveland, OH; Pamela B. Mangu, American Society of Clinical Oncology, Alexandria, VA; Manish A.

Shah, The Weill Cornell Medical Center; Philip A. Philip, Karmanos Cancer Institute, Detroit; Susan Urba, University of Michigan Cancer Center, Ann Arbor, MI; Eileen M. O'Reilly, Memorial Sloan Kettering Cancer Center; Joseph T. Ruggiero, Weill Cornell Medical College, New York, NY; Hope E. Uronis, Duke University, Durham, NC; Ramesh K. Ramanathan, Mayo Clinic, Scottsdale; Michelle Lau, Community Hospital Based Cancer Center, Tempe, AZ; Christopher $\mathrm{H}$. Crane, The University of Texas MD Anderson Cancer Center, Houston, TX; Anitra Engebretson, Patient Representative, Portland, OR; Mehmet S. Copur, St Francis Medical Center, Grand Island, NE; and Daniel Laheru, Johns Hopkins Sidney Kimmel Comprehensive Cancer Center, Baltimore, MD.

Published online ahead of print at www.jco.org on May 31, 2016.

D.P.S.S. and D.L. are co-chairs.

Clinical Practice Guidelines Committee approval: October 21, 2015.

Editor's note: This American Society of Clinical Oncology clinical practice

guideline provides recommendations with comprehensive review and analyses of the relevant literature for each recommendation. Additional information, which includes a Data Supplement with additional evidence tables, a Methodology Supplement, slide sets, clinical tools and resources, and links to patient information at www.cancer.net, is available at www. asco.org/guidelines/MetPC and www.asco. org/guidelineswiki.

Authors' disclosures of potential conflicts of interest and author contributions are found at the end of this article.

Reprint requests: American Society of Clinical Oncology, 2318 Mill Road, Suite 800, Alexandria, VA 22314; e-mail: guidelines@asco.org.

Corresponding author: American Society of Clinical Oncology, 2318 Mill Rd, Suite 800, Alexandria, VA 22314; e-mail:

guidelines@asco.org.

(C) 2016 by American Society of Clinical Oncology

0732-183X/16/3423w-2784w/\$20.00

DOI: 10.1200/JCO.2016.67.1412

\title{
Metastatic Pancreatic Cancer: American Society of Clinical Oncology Clinical Practice Guideline
}

Davendra P.S. Sohal, Pamela B. Mangu, Alok A. Khorana, Manish A. Shah, Philip A. Philip, Eileen M. O'Reilly, Hope E. Uronis, Ramesh K. Ramanathan, Christopher H. Crane, Anitra Engebretson, Joseph T. Ruggiero, Mehmet S. Copur, Michelle Lau, Susan Urba, and Daniel Laheru

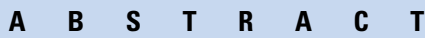

\section{Purpose}

To provide evidence-based recommendations to oncologists and others for the treatment of patients with metastatic pancreatic cancer.

\section{Methods}

American Society of Clinical Oncology convened an Expert Panel of medical oncology, radiation oncology, surgical oncology, gastroenterology, palliative care, and advocacy experts to conduct a systematic review of the literature from April 2004 to June 2015. Outcomes were overall survival, disease-free survival, progression-free survival, and adverse events.

\section{Results}

Twenty-four randomized controlled trials met the systematic review criteria.

\section{Recommendations}

A multiphase computed tomography scan of the chest, abdomen, and pelvis should be performed. Baseline performance status and comorbidity profile should be evaluated. Goals of care, patient preferences, treatment response, psychological status, support systems, and symptom burden should guide decisions for treatments. A palliative care referral should occur at first visit. FOLFIRINOX (leucovorin, fluorouracil, irinotecan, and oxaliplatin; favorable comorbidity profile) or gemcitabine plus nanoparticle albumin-bound (NAB) -paclitaxel (adequate comorbidity profile) should be offered to patients with Eastern Cooperative Oncology Group performance status (ECOG PS) 0 to 1 based on patient preference and support system available. Gemcitabine alone is recommended for patients with ECOG PS 2 or with a comorbidity profile that precludes other regimens; the addition of capecitabine or erlotinib may be offered. Patients with an ECOG PS $\geq 3$ and poorly controlled comorbid conditions should be offered cancer-directed therapy only on a case-by-case basis; supportive care should be emphasized. For second-line therapy, gemcitabine plus NABpaclitaxel should be offered to patients with first-line treatment with FOLFIRINOX, an ECOG PS 0 to 1 , and a favorable comorbidity profile; fluorouracil plus oxaliplatin, irinotecan, or nanoliposomal irinotecan should be offered to patients with first-line treatment with gemcitabine plus NABpaclitaxel, ECOG PS 0 to 1, and favorable comorbidity profile, and gemcitabine or fluorouracil should be offered to patients with either an ECOG PS 2 or a comorbidity profile that precludes other regimens. Additional information is available at www.asco.org/guidelines/MetPC and www.asco. org/guidelineswiki.

\section{J Clin Oncol 34:2784-2796. (C) 2016 by American Society of Clinical Oncology}

\section{INTRODUCTION}

Pancreatic ductal adenocarcinoma is a disease associated with poor prognosis and an increasing impact on cancer-related mortality in the United States and around the world. There were an estimated 49,000 new diagnoses and 41,000 deaths from pancreatic cancer in the United States in
$2015^{1}$ and an estimated 338,000 deaths worldwide in 2012. ${ }^{2}$ This disease remains an exception to the general trend of improvement in cancer-related mortality. One estimate suggests that pancreatic cancer will become the second leading cause of cancer-related death in the United States in the next decade. ${ }^{3}$ The 5 -year overall survival (OS) for metastatic pancreatic cancer remains at $2 \%,{ }^{4-6}$ with a median life expectancy of $<1$ year with current 


\section{Metastatic Pancreatic Cancer: American Society of Clinical Oncology Clinical Practice Guideline}

\section{Guideline Question}

What is the treatment of patients with metastatic pancreatic cancer?

\section{Target Population}

Patients with metastatic pancreatic cancer.

\section{Target Audience}

Medical oncologists, radiation oncologists, surgeons, gastroenterologists, and other caregivers

\section{Methods}

An Expert Panel developed clinical practice guideline recommendations that are based on a systematic review of the medical literature.

\section{Key Recommendations}

Recommendation 1.1: A multiphase CT scan of the chest, abdomen, and pelvis should be performed to assess extent of disease. Other staging studies should be performed only as dictated by symptoms (Type: evidence based, benefits outweigh harms; Evidence quality: intermediate; Strength of recommendation: strong).

Recommendation 1.2: The baseline PS, symptom burden, and comorbidity profile of a patient with metastatic pancreatic cancer should be evaluated carefully (Type: evidence based, benefits outweigh harms; Evidence quality: intermediate; Strength of recommendation: strong).

Recommendation 1.3: The goals of care (to include a discussion of an advance directive), patient preferences, as well as support systems should be discussed with every patient with metastatic pancreatic cancer and his or her caregivers (Type: evidence based, benefits outweigh harms; Evidence quality: intermediate; Strength of recommendation: strong).

Recommendation 1.4: Multidisciplinary collaboration to formulate treatment and care plans and disease management for patients with metastatic pancreatic cancer should be the standard of care (Type: evidence based, benefits outweigh harms; Evidence quality: intermediate; Strength of recommendation: strong).

Recommendation 1.5: Every patient with pancreatic cancer should be offered information about clinical trials, which include therapeutic trials in all lines of treatment as well as palliative care, biorepository/biomarker, and observational studies (Type: informal consensus, benefits outweigh harms; Evidence quality: intermediate; Strength of recommendation: strong).

Recommendation 2.1: FOLFIRINOX is recommended for patients who meet all of the following criteria: ECOG PS 0 to 1, favorable comorbidity profile, patient preference and support system for aggressive medical therapy, and access to chemotherapy port and infusion pump management services (Type: evidence based, benefits outweigh harms; Evidence quality: intermediate; Strength of recommendation: strong).

Recommendation 2.2: Gemcitabine plus NAB-paclitaxel is recommended for patients who meet all of the following criteria: ECOG PS 0 to 1, relatively favorable comorbidity profile, and patient preference and support system for relatively aggressive medical therapy (Type: evidence based, benefits outweigh harms; Evidence quality: intermediate; Strength of recommendation: strong).

Recommendation 2.3: Gemcitabine alone is recommended for patients who have either an ECOG PS 2 or a comorbidity profile that precludes more-aggressive regimens and who wish to pursue cancer-directed therapy. The addition of either capecitabine or erlotinib to gemcitabine may be offered in this setting (Type: evidence based, benefits outweigh harms; Evidence quality: intermediate; Strength of recommendation: moderate).

Recommendation 2.4: Patients with an ECOG PS $\geq 3$ or with poorly controlled comorbid conditions despite ongoing active medical care should be offered cancer-directed therapy only on a case-by-case basis. The major emphasis should be on optimizing supportive care measures (Type: evidence based, benefits outweigh harms; Evidence quality: intermediate; Strength of recommendation: moderate).

Recommendation 3.1: Gemcitabine plus NAB-paclitaxel can be offered as second-line therapy for patients who meet all of the following criteria: first-line treatment with FOLFIRINOX, ECOG PS 0 to 1, relatively favorable comorbidity profile, and patient preference and support system for aggressive medical therapy (Type: informal consensus, benefits outweigh harms; Evidence quality: low; Strength of recommendation: moderate).

(continued on following page) 
Recommendation 3.2: Fluorouracil plus oxaliplatin, irinotecan, or nanoliposomal irinotecan can be offered as second-line therapy for patients who meet all of the following criteria: first-line treatment with gemcitabine plus NAB-paclitaxel, ECOG PS 0 to 1, relatively favorable comorbidity profile, patient preference and support system for aggressive medical therapy, and chemotherapy port and infusion pump management (Type: informal consensus, benefits outweigh harms; Evidence quality: low; Strength of recommendation: moderate).

Recommendation 3.3: Gemcitabine or fluorouracil can be considered as second-line therapy for patients who have either an ECOG PS 2 or a comorbidity profile that precludes more-aggressive regimens and who wish to pursue cancer-directed therapy (Type: informal consensus, benefits outweigh harms; Evidence quality: low; Strength of recommendation: moderate).

Recommendation 3.4: No data are available to recommend third-line (or greater) therapy with a cytotoxic agent. Clinical trial participation is encouraged (Type: informal consensus, benefits outweigh harms; Evidence quality: low; Strength of recommendation: moderate).

Recommendation 4.1: Patients with metastatic pancreatic cancer should have a full assessment of symptom burden, psychological status, and social supports as early as possible, preferably at the first visit. In most cases, this assessment will indicate a need for a formal palliative care consult and services (Type: evidence based, benefits outweigh harms; Evidence quality: intermediate; Strength of recommendation: strong).

Recommendation 5.1: Patients with metastatic pancreatic cancer should be offered aggressive treatment of the pain and symptoms of the cancer and/or the cancer-directed therapy (Type: evidence based, benefits outweigh harms; Evidence quality: intermediate; Strength of recommendation: strong).

Recommendation 6.1: For patients on active cancer-directed therapy outside a clinical trial, imaging to assess first response should be offered at 2 to 3 months from the initiation of therapy. CT scans with contrast are the preferred modality. Thereafter, clinical assessment, conducted frequently during visits for cancer-directed therapy, should supplant imaging assessment. The routine use of positron emission tomography scans for the management of patients with pancreatic cancer is not recommended. CA19-9 is not considered an optimal substitute for imaging for the assessment of treatment response (Type: informal consensus, benefits outweigh harms; Evidence quality: low; Strength of recommendation: strong).

Recommendation 6.2: No data exist on the duration of cancer-directed therapy. An ongoing discussion of goals of care and assessment of treatment response and tolerability should guide decisions to continue or hold/terminate cancer-directed therapy (Type: informal consensus, benefits outweigh harms; Evidence quality: low; Strength of recommendation: strong).

\section{Additional Resources}

More information that includes a Data Supplement with additional evidence tables, a Methodology Supplement with information about evidence quality and strength of recommendations, slide sets, and clinical tools and resources is available at www.asco.org/ guidelines/MetPC and www.asco.org/guidelineswiki. Patient information is available at www.cancer.net

ASCO believes that cancer clinical trials are vital to inform medical decisions and improve cancer care and that all patients should have the opportunity to participate.

treatments. $^{7-9}$ Preclinical and clinical literature has established that pancreatic adenocarcinoma is a systemic disease from the outset, with early micrometastatic spread. ${ }^{10,11}$ Current multiagent chemotherapy regimens afford some gains in OS, albeit with attendant treatment-emergent toxicities. The clinical course of pancreatic cancer usually is aggressive, with high symptom burden and potential for a substantial deterioration in quality of life. These symptoms often include abdominal pain and loss of appetite, weight, and functional status. Other symptoms include biliary tract obstruction issues and pancreatic insufficiency, which lead to nutritional depletion. Therefore, palliative care to focus on distressing symptoms and quality of life is an important adjunct in the management of this condition.

The focus of this clinical practice guideline is to help with clinical decision making, which includes the determination of the appropriate treatment of patients with metastatic pancreatic cancer and how to help patients and their families to access and use palliative care services.

\section{GUIDELINE QUESTIONS}

This clinical practice guideline addresses six overarching clinical questions: After a histopathologic confirmation of pancreatic adenocarcinoma diagnosis, what initial assessment is recommended before initiating any therapy for metastatic pancreatic cancer? What is the appropriate first-line treatment of patients with metastatic pancreatic cancer? What is the appropriate therapy for patients with metastatic pancreatic cancer who experience either disease progression or intolerable toxicity with prior regimens for 
metastatic pancreatic cancer? When should the concept of palliative care be introduced? For patients with metastatic pancreatic cancer, what are the recommended strategies for relief of pain and symptoms? What is the recommended frequency of follow-up care/surveillance for patients with metastatic pancreatic cancer?

\section{METHODS}

\section{Guideline Development Process}

The Expert Panel met through webinar and teleconference and corresponded through e-mail. With consideration of the evidence, the authors contributed to the development of the guideline, provided critical review, and finalized the guideline recommendations. Members of the Expert Panel (Appendix Table A1, online only) were responsible for reviewing and approving the penultimate version of guideline, which was then circulated for external review and submitted to Journal of Clinical Oncology for editorial review and consideration for publication. All ASCO guidelines are ultimately reviewed and approved by the Expert Panel and the ASCO Clinical Practice Guidelines Committee before publication.

The recommendations were developed by the multidisciplinary Expert Panel using a systematic review of articles in English (April 2002 to June 2015) of phase III randomized controlled trials (RCTs) of chemotherapy alone and/or chemoradiotherapy and/or compared with a control arm. Other peer-reviewed articles were used to inform the recommendations on palliative care, patients with metastatic pancreatic cancer, and clinician communication as well as the section on health disparities. Articles were excluded from the systematic review if they were meeting abstracts not subsequently published in peer-reviewed journals; editorials, commentaries, letters, news articles, case reports, or narrative reviews; and published in a non-English language. The guideline recommendations were crafted, in part, by using the Guidelines Into Decision Support methodology and accompanying BRIDGE-Wiz software. ${ }^{12}$ In addition, a guideline implementability review was conducted. On the basis of the implementability review, revisions were made to the draft to clarify recommended actions for clinical practice. Ratings for the type and strength of recommendation, evidence, and potential bias are provided. In some selected cases where evidence is lacking but where there was a high level of agreement among the panel members, informal consensus was used (as noted in the recommendations).

Detailed information about the methods used to develop this guideline is available in the Methodology Supplement at www.asco.org/guidelines/ MetPC, which includes an overview (eg, panel composition, development process, revisions), literature search terms, and a data extraction quorum diagram; the recommendation development process (Guidelines Into Decision Support and BRIDGE-Wiz); and information about quality assessment.

The ASCO Expert Panel co-chairs and guidelines staff keep abreast of newly published data that signal an update to this guideline. Based on formal review of the emerging literature, ASCO staff will determine the need to update and post updates on www.asco.org/guidelines when indicated. The Methodology Supplement provides additional information about the Signals update approach.

This is the most recent information as of the publication date. Visit the ASCO Guidelines Wiki at www.asco.org/guidelineswiki to submit new evidence.

\section{Guideline Disclaimer}

The clinical practice guidelines and other guidance published herein are provided by the American Society of Clinical Oncology, Inc. (ASCO) to assist providers in clinical decision making. The information herein should not be relied upon as being complete or accurate, nor should it be considered as inclusive of all proper treatments or methods of care or as a statement of the standard of care. With the rapid development of scientific knowledge, new evidence may emerge between the time information is developed and when it is published or read. The information is not continually updated and may not reflect the most recent evidence. The information addresses only the topics specifically identified therein and is not applicable to other interventions, diseases, or stages of diseases. This information does not mandate any particular course of medical care. Further, the information is not intended to substitute for the independent professional judgment of the treating provider, as the information does not account for individual variation among patients. Recommendations reflect high, moderate, or low confidence that the recommendation reflects the net effect of a given course of action. The use of words like "must," "must not," "should," and "should not" indicates that a course of action is recommended or not recommended for either most or many patients, but there is latitude for the treating physician to select other courses of action in individual cases. In all cases, the selected course of action should be considered by the treating provider in the context of treating the individual patient. Use of the information is voluntary. ASCO provides this information on an "as is" basis and makes no warranty, express or implied, regarding the information. ASCO specifically disclaims any warranties of merchantability or fitness for a particular use or purpose. ASCO assumes no responsibility for any injury or damage to persons or property arising out of or related to any use of this information or for any errors or omissions.

\section{Guideline and Conflicts of Interest}

The Expert Panel was assembled in accordance with ASCO's Conflict of Interest Policy Implementation for Clinical Practice Guidelines ("Policy," found at http://www.asco.org/rwc). All members of the panel completed ASCO's disclosure form, which requires disclosure of financial and other interests, including relationships with commercial entities that are reasonably likely to experience direct regulatory or commercial impact as a result of promulgation of the guideline. Categories for disclosure include employment; leadership; stock or other ownership; honoraria, consulting or advisory role; speaker's bureau; research funding; patents, royalties, other intellectual property; expert testimony; travel, accommodations, expenses; and other relationships. In accordance with the Policy, the majority of the members of the panel did not disclose any relationships constituting a conflict under the Policy.

\section{RESULTS}

\section{Characteristics of Studies Identified in the Literature Search}

Twenty-five RCTs met the eligibility criteria and form the evidentiary basis for the guideline recommendations. ${ }^{8,9,13-35}$ The trials were generally of high quality, but few compared similar interventions. The primary outcome assessed for all included trials was therapeutic efficacy, which included OS and adverse events (AEs). Data Supplement 1, Table 1, lists the patient and disease characteristics of the studies pertinent to the development of the recommendations. Most studies were balanced for age and Eastern Cooperative Oncology Group performance status (ECOG PS). In all included trials, median age was younger (at least 5 years younger and for most, 10 years younger) than the median age of patients with pancreatic cancer in the general community. Previous treatments, if known, are also listed in the table.

\section{Study Quality Assessment}

Study design aspects related to individual study quality, strength of evidence, strength of recommendations, and risk of bias were 
assessed and are shown in Data Supplement 1, Table 2. The study quality was high for this group of RCTs. Design aspects related to the individual study quality were assessed with respect to factors such as blinding, allocation concealment, placebo control, intention to treat, funding sources, and so on, which generally indicated a low potential risk of bias for most of the identified evidence. Follow-up times varied among studies, which decreases the comparability of the results. Refer to Methodology Supplement for definitions of ratings for overall potential risk of bias.

\section{Key Outcomes of Interest}

Results for all outcomes of interest are response rates, OS, progression-free survival, disease-free survival, and AEs. Outcomes are included in Data Supplement 1, Table 3. The studies compared outcomes chemotherapy versus observation; chemotherapy versus chemoradiotherapy; and combination chemotherapy with or without radiotherapy for patients with metastatic pancreatic cancer.

\section{Systematic Reviews and Meta-Analyses for Potentially Curable Pancreatic Cancer}

Nine systematic reviews or meta-analyses of various rigor and quality were obtained. Because none were deemed suitable as the basis for recommendations, a formal assessment of quality was not performed. Data Supplement 2 is a summary table.

Data Supplements 3 and 4 include the literature review search terms and a quorum diagram of included and excluded articles. Data Supplement 5 contains information on the World Health Organization definition of palliative care, and Data Supplement 6 contains a pancreatic protocol for computerized tomography (CT).

\section{RECOMMENDATIONS}

\section{Clinical Question 1: After a Histopathologic Confirmation of Pancreatic Adenocarcinoma Diagnosis, What Initial Assessment Is Recommended Before Initiating Any Therapy for Metastatic Pancreatic Cancer?}

Recommendation 1.1. A multiphase CT scan of the chest, abdomen, and pelvis should be performed to assess extent of disease. Other staging studies should be performed only as dictated by symptoms (Type: evidence based, benefits outweigh harms; Evidence quality: intermediate; Strength of recommendation: strong).

Recommendation 1.2. The baseline PS, symptom burden, and comorbidity profile of a patient with metastatic pancreatic cancer should be evaluated carefully (Type: evidence based, benefits outweigh harms; Evidence quality: intermediate; Strength of recommendation: strong).

Recommendation 1.3. The goals of care (which include a discussion of an advance directive), patient preferences, and support systems should be discussed with every patient with metastatic pancreatic cancer and his or her caregivers (Type: evidence based, benefits outweigh harms; Evidence quality: intermediate; Strength of recommendation: strong).
Recommendation 1.4. Multidisciplinary collaboration to formulate treatment and care plans and disease management for patients with metastatic pancreatic cancer should be the standard of care (Type: evidence based, benefits outweigh harms; Evidence quality: intermediate; Strength of recommendation: strong).

Recommendation 1.5. Every patient with pancreatic cancer should be offered information about clinical trials, which include therapeutic trials in all lines of treatment as well as palliative care, biorepository/biomarker, and observational studies (Type: informal consensus, benefits outweigh harms; Evidence quality: intermediate; Strength of recommendation: strong).

Literature review and analysis. Cross-sectional imaging with a CT scan of the abdomen and pelvis by using a pancreatic protocol (Data Supplement 6) should be performed to evaluate the extent of disease in all patients with metastatic pancreatic cancer. Magnetic resonance imaging (MRI) appears to be of equivalent sensitivity to CTscanning with respect to its ability to detect and stage pancreatic cancer, but CT scanning is preferred because it is more easily interpreted and is less operator dependent. Similarly, acquisition and interpretation of echoendoscopy images is operator dependent; therefore, endoscopic ultrasound is most often used to facilitate acquisition of a biopsy specimen but not as a primary staging modality. A CT scan of the chest should be performed to evaluate for intrathoracic metastases.

Among patients with metastatic pancreatic cancer, baseline PS and a comorbidity profile should be evaluated thoroughly because both have implications with regard to a patient's ability to tolerate therapy. PS has been consistently identified as a prognostic factor for patients with pancreatic cancer. PS and comorbidities should not be used simply to rule in or out patients for treatment. For example, a patient with controlled diabetes mellitus or low hemoglobin levels, once optimized, could still do well with treatment because these comorbid conditions may be considered issues related to pancreatic disease.

After a comprehensive staging evaluation, a discussion on goals of care is important. This discussion should include the patient and key caregivers. Their understanding of the disease as well as treatment options, personal preferences, and social support systems should be addressed. On the basis of this mutual understanding of the goals of care, management decisions should be established within the context of a coordinated multidisciplinary group. If referral to a high-volume pancreatic cancer treatment center is feasible in a timely fashion, a referral should be offered because care at high-volume pancreatic treatment centers may lead to a change in therapeutic recommendations. ${ }^{36}$

Furthermore, enrollment in pancreatic cancer clinical trials should be encouraged. Currently, the accrual to such trials is suboptimal. In 2011 , only approximately $4.5 \%$ of patients with pancreatic cancer were enrolled onto a clinical trial, and only $14.9 \%$ of total anticipated enrollment in trials for patients with pancreatic cancer was achieved that year. ${ }^{37}$ Barriers to enrollment were need for travel, prohibitive illness, and physician opposition.

Clinical interpretation. The focus of the initial work-up should be to identify clues about both the extent of systemic disease and the ability to tolerate available therapies. The goals of therapy should be discussed clearly, with a focus on the palliative nature of any treatment plan and risks and benefits thereof. The available therapies may then be understood on the basis of the 
perceived goals of care. Management decisions should be made, as much as possible, in a multidisciplinary team environment. Clinical trials (therapeutic and otherwise) should be discussed with all patients.

\section{Clinical Question 2: What Is the Appropriate First-Line Treatment of Patients With Metastatic Pancreatic Cancer?}

Recommendation 2.1. Leucovorin, fluorouracil, irinotecan, and oxaliplatin (FOLFIRINOX) is recommended for patients who meet all of the following criteria: ECOG PS 0 to 1 , favorable comorbidity profile, patient preference and support system for aggressive medical therapy, and access to chemotherapy port and infusion pump management services (Type: evidence based, benefits outweigh harms; Evidence quality: intermediate; Strength of recommendation: strong).

Recommendation 2.2. Gemcitabine plus nanoparticle albuminbound (NAB) -paclitaxel is recommended for patients who meet all of the following criteria: ECOG PS 0 to 1, relatively favorable comorbidity profile, and patient preference and support system for relatively aggressive medical therapy (Type: evidence based, benefits outweigh harms; Evidence quality: intermediate; Strength of recommendation: strong).

Recommendation 2.3. Gemcitabine alone is recommended for patients who have either an ECOG PS 2 or a comorbidity profile that precludes more-aggressive regimens and who wish to pursue cancer-directed therapy. The addition of either capecitabine or erlotinib to gemcitabine may be offered in this setting (Type: evidence based, benefits outweigh harms; Evidence quality: intermediate; Strength of recommendation: moderate).

Recommendation 2.4. Patients with an ECOG PS $\geq 3$ or with poorly controlled comorbid conditions despite ongoing active medical care should be offered cancer-directed therapy on only a case-by-case basis. The major emphasis should be on optimizing supportive care measures (Type: evidence based, benefits outweigh harms; Evidence quality: intermediate; Strength of recommendation: moderate).

Literature review and analysis. FOLFIRINOX (fluorouracil $400 \mathrm{mg} / \mathrm{m}^{2}$ bolus, leucovorin $400 \mathrm{mg} / \mathrm{m}^{2}$, fluorouracil 2,400 mg/m over 46 hours, irinotecan $180 \mathrm{mg} / \mathrm{m}^{2}$, oxaliplatin $85 \mathrm{mg} / \mathrm{m}^{2}$, every 2 weeks) and gemcitabine plus NAB-paclitaxel (gemcitabine $1,000 \mathrm{mg} / \mathrm{m}^{2}$, NAB-paclitaxel $125 \mathrm{mg} / \mathrm{m}^{2}$, days $1,8,15$, every 4 weeks) are the two frontline regimens for metastatic pancreatic cancer management. Each regimen has been compared with gemcitabine, the previous standard of care, in large RCTs. ${ }^{8,9}$ However, no head-to-head comparisons of these two regimens exists. The FOLFIRINOX trial was conducted in France and enrolled 342 patients. The gemcitabine plus NAB-paclitaxel trial was conducted across many countries (North America, Europe, Australia) and enrolled 861 patients. Both trials had identical control arms: gemcitabine $1,000 \mathrm{mg} / \mathrm{m}^{2}$ given weekly for 7 or 8 weeks followed by administration on days 1, 8, 15, every 4 weeks. Key eligibility criteria for the FOLFIRINOX trial were metastatic disease, no prior chemotherapy, ECOG PS 0 or 1, bilirubin $\leq 1.5$ times the upper limit of normal, and age 18 to 75 years. Key eligibility criteria for the gemcitabine plus NAB-paclitaxel trial were metastatic disease, no prior chemotherapy (fluorouracil or gemcitabine as a radiation sensitizer $>6$ months before enrollment was allowed), Karnofsky performance status (KPS) of $\geq 70 \%$, bilirubin at or below the upper limit of normal, and no upper limit for age. Biliary stents were allowed in both trials if the bilirubin criterion was met. OS was the primary end point in each trial.

In the FOLFIRINOX trial, median OS with the experimental arm was 11.1 months compared with 6.8 months with gemcitabine (hazard ratio [HR], $0.57 ; 95 \% \mathrm{CI}, 0.45$ to $0.73 ; P<.001$ ). Response rate with FOLFIRINOX was $32 \%$. Major grade 3 or 4 toxicities with FOLFIRINOX were neutropenia (46\%), febrile neutropenia (5\%), fatigue $(24 \%)$, vomiting $(15 \%)$, diarrhea (13\%), and peripheral neuropathy $(9 \%)$. Growth factors were used in $43 \%$ of patients in the FOLFIRINOX arm.

In the gemcitabine plus NAB-paclitaxel trial, median OS with the experimental arm was 8.5 months compared with 6.7 months with gemcitabine (HR, $0.72 ; 95 \% \mathrm{CI}, 0.62$ to $0.83 ; P<.001$ ). Response rate with gemcitabine plus NAB-paclitaxel was $23 \%$. Major grade 3 or 4 toxicities with gemcitabine plus NAB-paclitaxel were neutropenia (38\%), febrile neutropenia (3\%), fatigue $(17 \%)$, diarrhea $(6 \%)$, and peripheral neuropathy $(17 \%)$. Growth factors were used in $26 \%$ of patients in the gemcitabine plus NABpaclitaxel arm.

In the absence of head-to-head comparisons of these two regimens in this setting, it is reasonable to offer either regimen. FOLFIRINOX is recommended in patients who meet all of the following criteria: ECOG PS 0 to 1, favorable comorbidity profile, patient preference and support system for aggressive medical therapy, and chemotherapy port and infusion pump management. A favorable comorbidity profile is loosely defined as hemoglobin $\geq 10 \mathrm{~g} / \mathrm{dL}$ and platelet count $\geq 100,000 / \mu \mathrm{L}$ without transfusion support; absolute neutrophil count $\geq 1,500 / \mu \mathrm{L}$; bilirubin and international normalized ratio $\leq 1.5$ times the upper limit of normal; albumin $\geq 3 \mathrm{~g} / \mathrm{dL}$; creatinine clearance $\geq 60 \mathrm{~mL} / \mathrm{min} /$ $1.73 \mathrm{~m}^{2}$; and absence of comorbid conditions that require ongoing active medical care, such as congestive heart failure, chronic obstructive pulmonary disease, uncontrolled diabetes mellitus, and neurologic disorders. Because FOLFIRINOX was not tested in patients older than 75 years, a carefully considered clinical decision to administer this regimen to anyone older than 75 years should be made.

Gemcitabine plus NAB-paclitaxel is recommended for patients who meet all of the following criteria: ECOG PS 0 to 1, relatively favorable comorbidity profile, and patient preference and support system for relatively aggressive medical therapy. A relatively favorable comorbidity profile is loosely defined as hemoglobin $\geq 9 \mathrm{~g} / \mathrm{dL}$ and platelet count $\geq 75,000 / \mu \mathrm{L}$ without transfusion support; absolute neutrophil count $\geq 1,500 / \mu \mathrm{L}$; bilirubin and international normalized ratio $\leq 1.5$ times the upper limit of normal; albumin $\geq 3 \mathrm{~g} / \mathrm{dL}$; creatinine clearance $\geq 60 \mathrm{~mL} / \mathrm{min} /$ $1.73 \mathrm{~m}^{2}$; and absence of poorly controlled comorbid conditions, such as congestive heart failure, chronic obstructive pulmonary disease, uncontrolled diabetes mellitus, and neurologic disorders. The gemcitabine plus NAB-paclitaxel trial allowed a KPS of 70\%, and $7 \%$ of patients in the study had a KPS of $70 \%$. Because the conversion of KPS and ECOG PS is not linear, ${ }^{38}$ a careful functional and comorbidity evaluation should be performed in patients with an ECOG PS 2 before offering gemcitabine plus NAB-paclitaxel. The comorbidity parameters here are only suggestions, and the treating 
clinician's judgment along with patient preferences are key factors to guide the choice between these two regimens.

Dose modifications are an important component of the ongoing treatment of patients with metastatic pancreatic cancer. At each visit (usually every 2 weeks), patients should be evaluated carefully for treatment-related toxicities, and these should be separated from disease-related symptoms. For treatment-related toxicities, doses should be reduced appropriately, preferably when toxicities are grade 2 or 3 , to prevent significant clinical worsening.

The following are suggestions based on dose-reduction schemes in the two clinical trials ${ }^{8,9}$ and early reports from institutional series of modified approaches to FOLFIRINOX and gemcitabine plus NAB-paclitaxel use. ${ }^{39,40}$ The treating physician's judgment remains paramount. For FOLFIRINOX, the omission of the fluorouracil bolus and leucovorin should be considered early (the omission of these from the first dose itself is not an unreasonable plan) in case of emerging toxicities. For hematologic toxicities, fatigue, nausea, and vomiting, dose reductions (usually by approximately 20\%) of irinotecan and oxaliplatin should be considered next. Further dose reductions for all three drugs should be considered for such ongoing toxicities. For diarrhea, dose reductions for irinotecan and fluorouracil should be considered, and for neuropathy, oxaliplatin dose should be reduced. Growth factor use to maintain blood counts is not routinely recommended for all patients. If blood count decline precludes chemotherapy administration, dose and/or drug modification should be the main therapeutic maneuver.

For gemcitabine plus NAB-paclitaxel, hematologic toxicities, fatigue, nausea, and vomiting, dose reductions (usually by approximately 20\%) for both drugs should be considered. For neuropathy, NAB-paclitaxel dose should be reduced. A commonly used maneuver with the use of this regimen is attenuation of schedule. Gemcitabine plus NAB-paclitaxel given at full doses but on days 1 and 15 of a 28-day cycle or on days 1 and 8 of a 21-day cycle are reasonable options to consider to mitigate treatment-related toxicities.

In patients who are not considered good candidates for FOLFIRINOX and gemcitabine plus NAB-paclitaxel due to comorbidities or who choose to pursue less-toxic therapies, gemcitabine $\left(1,000 \mathrm{mg} / \mathrm{m}^{2}\right.$, days $1,8,15$, every 4 weeks) is an appropriate option. Before data from the FOLFIRINOX and gemcitabine plus NAB-paclitaxel trials, this was the standard of care. ${ }^{41}$ The addition of either erlotinib or capecitabine to gemcitabine also can be offered, although data to support these recommendations are limited. Gemcitabine with erlotinib (gemcitabine $1,000 \mathrm{mg} / \mathrm{m}^{2}$, days $1,8,15$, every 4 weeks, with erlotinib 100 to $150 \mathrm{mg} /$ day orally) is approved in this setting. However, the added benefit of erlotinib is modest, with a nontrivial increase in treatment-associated toxicities and cost. ${ }^{26}$ Gemcitabine with capecitabine was tested in a clinical trial that showed a trend toward improvement of OS but did not meet the primary outcome. ${ }^{21}$

Clinical interpretation. FOLFIRINOX and gemcitabine plus NAB-paclitaxel are the two frontline regimens for the treatment of metastatic pancreatic cancer. In the absence of head-to-head comparisons, the choice depends on clinician judgment that is based on the patient's PS and comorbidities. Because the treatment is palliative, dose, drug, and schedule modifications should be incorporated liberally to maintain an appropriate risk-benefit balance. Gemcitabine, either alone or with erlotinib, is another available option, albeit limited to patients who are assessed as having a PS that is not robust enough to handle multiagent cytotoxic regimens but who still wish to pursue cancer-directed therapy.

\section{Clinical Question 3: What Is the Appropriate Therapy for Patients With Metastatic Pancreatic Cancer Who Experience Either Disease Progression or Intolerable Toxicity With Prior Regimens for Metastatic Pancreatic Cancer?}

Recommendation 3.1. Gemcitabine plus NAB-paclitaxel can be offered as second-line therapy for patients who meet all of the following criteria: first-line treatment with FOLFIRINOX, ECOG PS 0 to 1 , relatively favorable comorbidity profile, and patient preference and a support system for aggressive medical therapy (Type: informal consensus, benefits outweigh harms; Evidence quality: low; Strength of recommendation: moderate).

Recommendation 3.2. Fluorouracil plus oxaliplatin, irinotecan, or nanoliposomal irinotecan can be offered as second-line therapy for patients who meet all of the following criteria: first-line treatment with gemcitabine plus NAB-paclitaxel, ECOG PS 0 to 1, relatively favorable comorbidity profile, patient preference and a support system for aggressive medical therapy, and chemotherapy port and infusion pump management (Type: informal consensus, benefits outweigh harms; Evidence quality: low; Strength of recommendation: moderate).

Recommendation 3.3. Gemcitabine or fluorouracil can be considered as second-line therapy for patients who have either an ECOG PS of 2 or a comorbidity profile that precludes moreaggressive regimens and who wish to pursue cancer-directed therapy (Type: informal consensus, benefits outweigh harms; Evidence quality: low; Strength of recommendation: moderate).

Recommendation 3.4. No data are available to recommend thirdline (or greater) therapy with a cytotoxic agent. Clinical trial participation is encouraged (Type: informal consensus, benefits outweigh harms; Evidence quality: low; Strength of recommendation: moderate).

Literature review and analysis. No prospective studies have evaluated second-line therapy for metastatic pancreatic cancer that has progressed on either FOLFIRINOX or gemcitabine plus NABpaclitaxel. The only available second-line data are from trials where metastatic pancreatic cancer progressed on first-line gemcitabine. The CONKO-003 trial, which tested fluorouracil plus oxaliplatin (leucovorin $200 \mathrm{mg} / \mathrm{m}^{2}$ and fluorouracil 2,000 $\mathrm{mg} / \mathrm{m}^{2}$ over 24 hours on days $1,8,15$, and 22 with oxaliplatin $85 \mathrm{mg} / \mathrm{m}^{2}$ on days 8 and 22) against fluorouracil in metastatic pancreatic cancer that had progressed on first-line gemcitabine, ${ }^{13}$ showed improved OS with the combination ( $5.9 v 3.3$ months; HR, 0.66 ; $95 \%$ CI, 0.48 to $0.91 ; P=.01$ ). The NAPOLI-1 trial, which tested fluorouracil plus nanoliposomal irinotecan (nanoliposomal irinotecan $80 \mathrm{mg} / \mathrm{m}^{2}$, leucovorin $400 \mathrm{mg} / \mathrm{m}^{2}$, and fluorouracil 2,400 mg/m ${ }^{2}$ over 46 hours every 2 weeks) against fluorouracil in metastatic pancreatic cancer that had progressed on first-line gemcitabine, showed improved OS with the combination (6.1 $v 4.2$ months; HR, 0.67; $95 \% \mathrm{CI}, 0.49$ to $0.92 ; P=.01){ }^{40}$ A meta-analysis of clinical trials comparing second-line chemotherapy with best supportive care alone showed that median OS was 6 months with chemotherapy compared with 2.8 months with best supportive care. ${ }^{42}$ Because most patients now receive multiagent regimens instead of gemcitabine in the first-line setting, the CONKO-003 and 
NAPOLI-1 results do not apply to most clinical situations. However, the panel arrived at a consensus that after progression on (or intolerable toxicity from) a first-line regimen of FOLFIRINOX or gemcitabine plus NAB-paclitaxel, second-line chemotherapy should provide clinical benefit. The choice of a second-line regimen is not defined; it is reasonable to offer drugs that the patient has not been exposed to in the first-line regimen. Gemcitabine plus NABpaclitaxel (gemcitabine 1,000 mg/m², NAB-paclitaxel $125 \mathrm{mg} / \mathrm{m}^{2}$, days $1,8,15$, every 4 weeks) can be offered as second-line therapy to persons who received FOLFIRINOX in the first-line setting and maintain a preserved comorbidity profile and a patient preference and support system for aggressive medical therapy. In case of residual toxicities from FOLFIRINOX, it is reasonable to start therapy at attenuated doses and/or schedules. Gemcitabine $800 \mathrm{mg} / \mathrm{m}^{2}$, NAB-paclitaxel $100 \mathrm{mg} / \mathrm{m}^{2}$ on days 1 and 8 every 3 weeks are some alternatives to consider. Similarly, fluorouracil plus oxaliplatin, irinotecan, or nanoliposomal irinotecan can be offered as secondline therapy to patients who received gemcitabine plus NABpaclitaxel in the first-line setting and maintained a preserved comorbidity profile and who have a preference and support system for aggressive medical therapy. It is reasonable to omit fluorouracil bolus and leucovorin in this setting.

Clinical interpretation. No good data exist for the second-line (or greater) treatment of patients with metastatic pancreatic cancer that has progressed on contemporary first-line regimens such as FOLFIRINOX and gemcitabine plus NAB-paclitaxel. Extrapolation from clinical trials in patients with metastatic pancreatic cancer that progressed on first-line gemcitabine leads to a reasonable conclusion that OS can be improved with second-line cytotoxic therapy. The choice of the agents depends on patient PS, comorbidities, organ function, and residual toxicities from the first-line regimen.

\section{Clinical Question 4: When Should the Concept of Palliative Care Be Introduced?}

Recommendation 4.1. Patients with metastatic pancreatic cancer should have a full assessment of symptom burden, psychological status, and social supports as early as possible, preferably at the first visit. In most cases, this assessment will indicate a need for a formal palliative care consult and services (Type: evidence based, benefits outweigh harms; Evidence quality: intermediate; Strength of recommendation: strong).

Literature review and analysis. Patients with metastatic pancreatic cancer tend to have a high symptom burden at the time of diagnosis, although this varies with the extent of disease. A full assessment of symptoms to include psychological status is recommended. Social supports should be ascertained as well during the first visit. If available, a formal palliative care consult can introduce the patient to the full range of services available to assure that close attention will be paid to physical comfort, pain management, psychosocial concerns, and spiritual well-being throughout the trajectory of the illness. Palliative care, in its broadest definition, is the supportive care of a patient and family from diagnosis through treatment (either curative or noncurative) until death. Hospice care is a subset of palliative care focused on patients near the end of life. A survey showed that nearly $90 \%$ of adults in the United States had no knowledge or limited knowledge of palliative care services. When they were read a definition, $>90 \%$ of the respondents said that they would want palliative care for themselves or their family member and that it should be universally available. $^{43,44}$ If the patient presents with extensive disease, is too ill to tolerate treatment, or has progressive disease for which there is no reasonable further anticancer treatment, then a hospice discussion and possible referral should take place.

\section{Clinical Question 5: For Patients With Metastatic Pancreatic Cancer, What Are the Recommended Strategies for Relief of Pain and Symptoms?}

Recommendation 5.1. Patients with metastatic pancreatic cancer should be offered aggressive treatment of the pain and symptoms of the cancer and/or the cancer-directed therapy (Type: evidence based, benefits outweigh harms; Evidence quality: intermediate; Strength of recommendation: strong).

Literature review and analysis. Patients with pancreatic cancer may experience various distressing symptoms and concerns that require ongoing supportive care.

Pain. The mainstay of pain management typically is opiate medication, and physicians must address the level of pain and the degree of pain relief from analgesics at every clinic visit. Initial pain management may involve nonopioid drugs, which includes paracetamol. Disease progression may require the use of stronger opioids (tramadol, morphine, or fentanyl). ${ }^{45}$ Because of the proximity of the tumor to the celiac axis, the pain may be neuropathic in nature. This would warrant consideration of treatment with adjuvant medications such as gabapentin, pregabalin, nortriptyline, and duloxetine.

Adverse effects and decreased effectiveness may limit the use of medications, in which case, treatments to interrupt the neural pathways in the celiac plexus may be used to improve pain relief. The celiac plexus is a dense network of nerves that innervates the upper abdominal organs and varies in structure across individuals. Pain may be relieved by inhibiting these synaptic pathways without neuron destruction in celiac plexus block through surgery with thoracoscopic splanchinectomy, which interrupts the parasympathetic and sympathetic fibers in the celiac plexus, or chemical destruction of the pathways and ganglia with dehydrated alcohol through celiac plexus neurolysis. ${ }^{45}$

One hundred patients with unresectable pancreatic cancer who experienced pain were randomly assigned to receive either neurolytic celiac plexus block or systemic analgesic therapy. ${ }^{46}$ The group treated with the neurolytic block had a larger initial decrease in pain $(P=.005)$, and the improvement effect lasted over time. Another RCT was conducted in 109 patients with inoperable abdominal or pelvic cancer, 38 of whom had pancreatic cancer. ${ }^{47}$ The trial evaluated the timing of neurolytic sympathectomy, performed either early after the diagnosis of the pain or later in the patient's course after not obtaining pain relief with strong opioids. Early sympathectomy led to better pain control, less opioid consumption, and better quality of life in these patients. Palliative radiotherapy or chemotherapy may be considered to augment pain management.

Anorexia, weight loss. Patients merit a consultation with a nutritionist and/or dietician if this service is available. Dietary intake can be assessed along with the possible need for nutritional 
supplements. Some patients experience exocrine pancreatic insufficiency and require pancreatic enzyme replacement. Pancrelipase replacement daily with meals can help to improve digestion and absorption of nutrients. A placebo-controlled, double-blind trial of enteric-coated pancreatin microspheres was conducted in patients with unresectable cancer in the pancreatic head. Patients on pancreatic enzymes along with dietary counseling gained $1.2 \%(0.7 \mathrm{~kg})$ body weight, whereas patients on placebo lost $3.7 \%$ body weight $(2.2 \mathrm{~kg}){ }^{48,49}$ Appetite stimulant medications may be considered in severe cases.

Depression and anxiety. The diagnosis of cancer is unsettling to any patient, and the knowledge of the aggressive nature of metastatic pancreatic cancer may lead to depression or anxiety, even early in the course of the disease. All patients can benefit from a discussion of their psychosocial concerns and their available support system. Some may warrant treatment with antidepressants or anxiolytics, and others may need referral for ongoing formal support from a social worker or psychiatrist.

Biliary obstruction. A frequent complication of a pancreatic tumor is blockage of the biliary tree, which causes obstructive jaundice. The preferred treatment is endoscopic placement of a permanent self-expanding stent in the bile duct to re-establish drainage to achieve relief of jaundice and pruritus; normalization of bilirubin levels to allow palliative chemotherapy; and prevention of other adverse outcomes, such as cholangitis and frequent hospitalizations. ${ }^{50}$ The choice of stent depends on patient prognosis and the relative costs of metal stents and repeat endoscopic retrograde cholangiopancreatographies. In general, metal stents are preferred. Plastic stents can be considered for patients expected to survive $<3$ months. ${ }^{51}$

Gastric outlet/duodenal obstruction. Gastric outlet/duodenal obstruction occurs in up to $10 \%$ of patients with pancreatic cancer. Symptoms include early satiety, nausea, postprandial vomiting, and weight loss. Endoscopic duodenal stenting can be successful in the majority of these patients, and median duration of stent patency is 6 months. $^{52}$

Ascites. Patients with malignant ascites from pancreatic cancer experience abdominal discomfort, nausea and vomiting, and dyspnea from pressure of the fluid against the diaphragm. Many will benefit from intermittent paracentesis for symptom relief, but unfortunately, the benefit often lasts only approximately 3 days and then must be repeated. Diuretics such as spironolactone are competitive antagonists to aldosterone; they decrease the reabsorption of water and sodium in the kidneys and provide some relief from ascites.

If ascites reaccumulate quickly, which requires frequent (more than once a week or so) paracentesis, placement of a long-term drainage catheter is suitable. Circulating blood volume is reduced and the renin-angiotensin-aldosterone system is activated, which lead to sodium retention.

Venous thromboembolism. The occurrence of deep venous thrombosis, pulmonary embolism, and visceral vein thrombi (eg, portal vein, superior mesenteric vein thrombus) is extremely prevalent in patients with pancreatic cancer. Indeed, in most epidemiologic studies, pancreatic cancer ranks as one of the malignancies with the highest incidence of venous thromboembolism (VTE). This may be driven by the early expression of tissue factor on preneoplastic and neoplastic pancreas. ${ }^{53}$ The development of VTE is highly consequential to patients with cancer. It is associated with worsened short- and long-term mortality ${ }^{54}$ and is the second leading cause of death in malignancy after the cancer itself. ${ }^{55}$ Unfortunately, patients with cancer remain unaware of this complication and its treatments. As recommended by the ASCO guidelines on VTE, patients need to be educated on the warning signs and symptoms of VTE. ${ }^{56}$ Primary prevention of VTE can be successfully achieved with the use of low-molecular-weight heparins (LMWHs). Two RCTs have addressed the utility of primary prophylaxis with LMWH in patients with advanced pancreatic cancer, and all have shown a substantial reduction of VTE. ${ }^{57,58}$ Concordant with ASCO guidelines on VTE, ${ }^{56}$ the panel recommends consideration of primary prophylaxis in select high-risk patients on a case-by-case basis while they undergo systemic therapy. Treatment of pancreatic cancer-associated VTE is best achieved with extended LMWH monotherapy. The utility of treatment of incidentally identified visceral vein thrombi is unclear; the decision to anticoagulate can be made on a case-by-case basis.

Clinical interpretation. Refer to other ASCO guidelines and other evidence-based guidelines (eg, VTE, peripheral neuropathy, fatigue, anxiety and depression, antiemetics, prophylaxis and management of fever and neutropenia, WBC growth factors) for more detailed information in the patient and survivor care and supportive care and treatment-related issues sections at www.asco.org/guidelines.

\section{Clinical Question 6: What Is the Recommended Frequency of Follow-Up Care/Surveillance for Patients With Metastatic Pancreatic Cancer?}

Recommendation 6.1. For patients on active cancer-directed therapy outside a clinical trial, imaging to assess first response should be offered at 2 to 3 months from the initiation of therapy. CT scans with contrast are the preferred modality. Thereafter, clinical assessment conducted frequently during visits for cancer-directed therapy should supplant imaging assessment. The routine use of positron emission tomography scans for the management of patients with pancreatic cancer is not recommended. CA19-9 is not considered an optimal substitute for imaging for assessing treatment response (Type: informal consensus, benefits outweigh harms; Evidence quality: low; Strength of recommendation: strong).

Recommendation 6.2. No data exist on the duration of cancerdirected therapy. An ongoing discussion of goals of care and assessment of treatment response and tolerability should guide decisions to continue or hold/terminate cancer-directed therapy (Type: informal consensus, benefits outweigh harms; Evidence quality: low; Strength of recommendation: strong).

Literature review and analysis. At present, no evidence-based data exist to guide the frequency of imaging for patients with metastatic cancer. The two approved chemotherapy combinations of FOLFIRINOX (once every 2 weeks) and gemcitabine plus NAB-paclitaxel (days 1, 8, 15 every 4 weeks) lend themselves naturally to follow-up imaging after 8 or 12 weeks of chemotherapy. The clinical practice for physicians in this panel would be to reimage after 8 to 12 weeks of chemotherapy.

\section{PATIENT AND CLINICIAN COMMUNICATION}

This section is based on experience and selected literature but was not part of the systematic review of the literature. Patients with 
pancreatic cancer face difficult treatment decisions while presented with complex medical information and a life-threatening diagnosis. Communication within a context of realistic hope and action between patients and clinicians can improve patients' ability to make sound, informed decisions within their own personal value set. ${ }^{59}$ Patients should fully understand goals of care before making decisions about treatment and care.

Clear communication with patients with pancreatic cancer and their caregivers about the diagnosis, treatment options, and goals of care is key for patient understanding. The clinician is also responsible for offering ancillary support services, which include a referral to a palliative care consultation and services.

For patients to make informed decisions, providers should describe the potential impact of the diagnosis of pancreatic cancer on the patient and family. It is important to provide realistic hope within honest, yet supportive discussions. Providers should ask patients about their personal goals and preferences. What do they hope for? What is important to them in their personal lives? What do they value more, an extension of life or maintenance of the best possible quality of life? An understanding of a patient's specific goals should shape conversations about goals of care and treatment recommendations.

Clinicians should clearly explain all potential treatment options, the potential outcomes of each, and possible AEs/adverse effects so that patients understand benefits and drawbacks of each and can make an informed decision. Treatment discussions should include relevant clinical trials at every stage of treatment. Patients should have the opportunity to participate in trials for their own treatment as well as be given the opportunity to contribute to research.

Clinicians should also consider and proactively discuss quality-of-life issues. In patients with pancreatic cancer, dietary concerns, pain, and fatigue are major concerns. Dietary issues tend to be overlooked and yet are real problems with a significant impact on daily life. Referral to a registered dietitian and/or gastroenterologist with early intervention can be of great benefit. Clinicians should also consider the use of and discuss the possible need for pancreatic enzyme replacement therapy.

Referral to palliative care services can facilitate the addressing of many non-treatment-related issues patients face and should be offered for all patients with pancreatic cancer, regardless of stage of disease or expected prognosis. Patients should understand that referral to a consultation for palliative care services is not synonymous with a referral to hospice care. This discussion is important because palliative care provides important support and can be part of an active cancer treatment paradigm.

Patients must feel comfortable in the choices they make, and the knowledge that they have explored their options can bring comfort. ${ }^{59}$ As such, clinicians should support a patient's desire to get a second opinion. Clinicians should address the costs of care and offer referrals to specialists within the health care system who can discuss in more detail what a patient should expect as well as resources and information about managing the costs related to cancer care.

The provision of realistic hope to patients with pancreatic cancer, although the prognosis may be short, is important. Patients deserve to know that their medical team is working to help them reach their goals. Even if a cure is not possible, hope for an extension of life or good quality of life is powerful.

The provision of resources to help patients communicate better with their health care team is also advisable. Patients should be offered decision-making tools and urged to write down questions in between and in advance of appointments. Patients can be referred to resources that will extend the support and information clinicians are able to provide. For pancreatic cancer, two such resources are the ASCO patient-facing Web site (www.Cancer.net) and the Pancreatic Cancer Action Network (www.pancan.org).

\section{Metastatic Pancreatic Cancer}

- Explain all potential treatment options and possible AEs/ adverse effects, which include clinical trials, so that the patient understands benefits and drawbacks of each and can make an informed decision.

- Discuss that a referral for a consultation with palliative care services does not mean hospice; it is in conjunction with active treatment.

- Ask about patient preferences and personal goals of care.

- Understand that patients may want active treatment to shrink or hold back cancer, extend life, and/or reduce pain and adverse effects

- Offer patients and their families a tool to discuss options (a decision aid to be used in conjunction with clinicians).

- Urge patients to write down questions to ask the clinician on follow-up visits. Support a second opinion by urging patients to consider high-volume centers for treatment of metastatic pancreatic cancer.

- List resources and support (ie, Pancreatic Cancer Action Network, Cancer.net).

\section{HEALTH DISPARITIES}

Although ASCO clinical practice guidelines represent expert recommendations on best practices in disease management to provide the highest level of cancer care, many people have limited access to medical care. Racial and ethnic disparities in health care contribute significantly to this problem in the United States. People with cancer who are members of racial/ethnic minorities suffer disproportionately from comorbidities, experience more substantial obstacles to receiving care, are more likely to be uninsured, and are at greater risk of receiving poor quality care than other Americans. ${ }^{60}$ Many other people lack access to care because of their geographic location and distance from appropriate treatment facilities. Awareness of these disparities in access to care should be considered in the context of this clinical practice guideline, and health care providers should strive to deliver the highest level of cancer care to these vulnerable populations.

Such disparities may not exist in a more equal-access system. By using the Department of Defense tumor registry database from 1993 to 2007, patient, tumor, and treatment factors were analyzed to compare rates of therapy and survival between blacks and whites. ${ }^{61}$ Of 1,008 patients with pancreatic cancer, 157 (15\%) were black. Thirty-six percent of black and $37 \%$ of white patients presented with locoregional disease $(P=.85)$. For example, among those with potentially curable cancers, the odds of black patients having received surgical resection (odds ratio [OR], 1.06; 95\% CI, 0.60 to 1.89 ), chemotherapy (OR, $0.92 ; 95 \% \mathrm{CI}, 0.49$ to 1.73 ), and radiotherapy (OR, 1.14; 95\% CI, 0.61 to 2.10 ) were not different 
from those of whites. Among those with distant disease, the odds of having received palliative chemotherapy were also similar (OR, $0.91 ; 95 \% \mathrm{CI}, 0.55$ to 1.51 ). In a multivariable analysis, black race compared with white race was not associated with shorter OS. These data suggest that improvement in the access to health care of minorities with pancreatic cancer may reduce disparities in oncologic outcomes. Thus, a significant proportion of patients with pancreatic cancer remain undertreated, possibly as a result of two nonclinical factors such as insurance status and access to care.

\section{MULTIPLE CHRONIC CONDITIONS}

The creation of evidence-based recommendations to inform treatment of patients with additional chronic conditions, a situation in which a person may have two or more such conditions (referred to as multiple chronic conditions [MCCs]), is challenging. Even in clinical trials that enroll highly selected patients with metastatic pancreatic cancer, tolerance and completion of adjuvant therapy are challenging due to AEs and toxicities. ${ }^{62}$ Patients who have health status considerations that would have precluded them from participation in the clinical trials that establish the evidence may have uncertain benefit from interventions for their cancer, and this uncertainty should be discussed with the patient during the informed consent process for treatment. Older patients, for example, who have other health status conditions are at higher risk for chemotherapy toxicity. ${ }^{63}$ In patients with metastatic pancreatic cancer, these risks should be assessed and discussed, especially given that first-line treatments have a high prevalence of toxicity, and clinical trials have not included patients with significant MCCs or those at advanced ages.

Patients with metastatic pancreatic cancer with MCCs are a complex and heterogeneous population, which makes it difficult to account for all the possible permutations to develop specific recommendations for care. In addition, the best available evidence for treating index conditions, such as cancer, is often from clinical trials wherein study selection criteria may have excluded patients to avoid potential interaction effects or confounding of results associated with MCCs. As a result, the reliability of outcome data from these studies may be limited, thereby creating constraints for expert groups to make recommendations for care in this heterogeneous population.

Because many patients with pancreatic cancer for whom guideline recommendations apply present with MCCs, any treatment plan needs to take into account the complexity and uncertainty created by the presence of MCCs and highlight the importance of shared decision making with regard to guideline use and implementation. Therefore, in consideration of recommended care for the target index condition, clinicians should review all other chronic conditions present in patients with metastatic pancreatic cancer and take those conditions into account when formulating the treatment and follow-up plan.

\section{COST IMPLICATIONS}

Limited cost-effectiveness analyses exist with regard to the various treatment modalities used in the multidisciplinary management of metastatic pancreatic cancer. However, the available data appear to support the recommendations in this guideline. One study assessed the cost-effectiveness of first-line FOLFIRINOX compared with first-line gemcitabine for public payers in Canada. Compared with first-line gemcitabine, first-line FOLFIRINOX resulted in more life-years and quality-adjusted life-years. Probabilistic sensitivity analysis results showed that for analyses 1 and 2, respectively, FOLFIRINOX has a $>85 \%$ probability and an approximately $80 \%$ probability of being cost-effective at the $\$ 100,000$ threshold. Compared with gemcitabine, first-line FOLFIRINOX significantly prolongs median OS. Given the favorable cost per quality-adjusted life-year, the improvement in clinical efficacy, and the limited available treatment options, FOLFIRINOX represents an attractive cost-effective treatment. ${ }^{64}$

As reported at the 2014 Gastrointestinal Cancers Symposium, investigators compared the costs and clinical outcomes of gemcitabine plus NAB-paclitaxel versus erlotinib plus gemcitabine $(\mathrm{E} / \mathrm{G})$ by using drug cost per cycle multiplied by the median cycles delivered from clinical trials for gemcitabine plus NAB-paclitaxel and $\mathrm{E} / \mathrm{G}$. The comparison included the cost of the drugs as well as expenses related to the administration of the therapy and the management of AEs of grade 3/4 severity. These costs were based on 4 months of therapy for gemcitabine plus NAB-paclitaxel versus 3.9 months for $\mathrm{E} / \mathrm{G}$ as administered at a large, multisite oncology clinic. The researchers found that the total cost for gemcitabine plus NAB paclitaxel was $\$ 24,984$ versus $\$ 23,044$ for E/G. However, the gemcitabine plus NAB-paclitaxel is expected to deliver a greater survival benefit based on clinical trial data, bringing the cost per life-year gained to $\$ 15,522 .^{65}$

Moreover, health care experts have noted that the costs of treatment are high and increasing. The choice of therapy depends on a variety of clinical factors. More than $70 \%$ of cases are diagnosed in patients age 65 years and older. Thus, in the United States, Medicare pays for a substantial portion of associated costs. The costs of treating the malignancy are noteworthy when one considers that pancreatic cancer is the fourth leading cause of cancer-related mortality in the United States.

\section{EXTERNAL REVIEW}

The draft was submitted to two external reviewers with content expertise. It was rated as high quality, and it was agreed that the guideline would be useful in practice. The Expert Panel reviewed the comments and integrated them into the final manuscript before approval by the Clinical Practice Guidelines Committee.

\section{GUIDELINE IMPLEMENTATION}

ASCO guidelines are developed for implementation across health settings. Barriers to implementation include the need to increase awareness of the guideline recommendations among frontline practitioners, cancer survivors, and caregivers and to provide adequate services in the face of limited resources. The guideline Bottom Line Box was designed to facilitate implementation of recommendations. This guideline will be distributed widely through the ASCO Practice Guideline Implementation Network. ASCO guidelines are posted on the ASCO Web site and most often 
published in Journal of Clinical Oncology and Journal of Oncology Practice.

\section{LIMITATION OF THE RESEARCH AND FUTURE DIRECTIONS}

Many research initiatives are aimed at improving the diagnosis and treatment of metastatic pancreatic cancer. Groups collaborate to find treatments and improve screening and diagnosis with biomarkers of pancreatic cancer, which could help physicians to diagnose the disease earlier and provide better treatment to patients with pancreatic cancer.

A prospective trial conducted through the National Cancer Institute National Clinical Trials Network that compares a treatment regimen that contains the novel agent NAB-paclitaxel with more established cytotoxic agents (fluorouracil, oxaliplatin, and irinotecan) may address the question of optimal first-line treatment of metastatic pancreatic cancer and determine the feasibility and impact of second-line therapy. A large phase III trial with smaller pilot and phase II trials to study novel interventions would be possible because of the large number of patients with pancreatic cancer. The question then remains about whether this large-scale study to determine superiority should be prioritized or whether resources would be better used toward another strategy, especially when many patients will receive both regimens throughout their treatment. $^{66}$

Other strategies that would de-emphasize the treatment of all cases of metastatic pancreatic cancer with the same intervention should also be explored. An investigation of the molecular genetics and biology of pancreatic cancers would be more effective to identify subsets that would respond to single agents or combinations of targeted agents or a cytotoxic backbone. Also of value would be to explore immunologic approaches, such as CD40 agonists and T-cell engineering. ${ }^{66}$ Each of these options recognizes the individual variation among patients with metastatic pancreatic cancer and allows for individualized treatments.

ASCO believes that cancer clinical trials are vital to inform medical decisions and improve cancer care and that all patients should have the opportunity to participate.

\section{ADDITIONAL RESOURCES}

More information, which includes a Data Supplement with additional evidence tables, a Methodology Supplement with information about evidence quality and strength of recommendations, slide sets, and other clinical tools and resources, are available at www.asco.org/ guidelines/MetPC. Patient information is available at www.cancer.net. Visit www.asco.org/guidelineswiki to provide comments on the guideline or to submit new evidence.

\section{AUTHORS' DISCLOSURES OF POTENTIAL CONFLICTS OF INTEREST}

Disclosures provided by the authors are available with this article at www.jco.org.

\section{AUTHOR CONTRIBUTIONS}

Administrative support: Pamela B. Mangu

Manuscript writing: All authors

Final approval of manuscript: All authors

\section{REFERENCES}

1. American Cancer Society: Cancer facts \& figures 2015. www.cancer.org/acs/groups/content/@ editorial/documents/document/acspc-044552.pdf

2. World Cancer Research Fund International: Pancreatic cancer statistics. www.wcrf.org/int/cancerfacts-figures/data-specific-cancers/pancreatic-cancerstatistics

3. Rahib L, Smith BD, Aizenberg R, et al: Projecting cancer incidence and deaths to 2030: The unexpected burden of thyroid, liver, and pancreas cancers in the United States. Cancer Res 74:2913-2921, 2014

4. Jemal $A$, Siegel $R$, Ward $E$, et al: Cancer statistics, 2006. CA Cancer J Clin 56:106-130, 2006

5. American Cancer Society: Cancer facts \& figures 2013. www.cancer.org/research/cancerfactsfigures/ cancerfactsfigures/cancer-facts-figures-2013

6. Malvezzi M, Bertuccio P, Levi F, et al: European cancer mortality predictions for the year 2013. Ann Oncol 24:792-800, 2013

7. Hawes $\mathrm{RH}$, Xiong $\mathrm{Q}$, Waxman I, et al: $\mathrm{A}$ multispecialty approach to the diagnosis and management of pancreatic cancer. Am J Gastroenterol 95:17-31, 2000

8. Conroy $T$, Desseigne $F$, Ychou $M$, et al: FOLFIRINOX versus gemcitabine for metastatic pancreatic cancer. N Engl J Med 364:1817-1825, 2011

9. Von Hoff DD, Ervin $T$, Arena FP, et al: Increased survival in pancreatic cancer with nabpaclitaxel plus gemcitabine. N Engl J Med 369: 1691-1703, 2013

10. Sohal DP, Walsh RM, Ramanathan $R K$, et al: Pancreatic adenocarcinoma: Treating a systemic disease with systemic therapy. J Natl Cancer Inst 106:dju011, 2014

11. Rhim AD, Mirek ET, Aiello NM, et al: EMT and dissemination precede pancreatic tumor formation. Cell 148:349-361, 2012

12. Shiffman RN, Michel G, Rosenfeld RM, et al: Building better guidelines with BRIDGE-Wiz: Development and evaluation of a software assistant to promote clarity, transparency, and implementability. J Am Med Inform Assoc 19:94-101, 2012

13. Oettle H, Riess H, Stieler JM et al: Second-line oxaliplatin, folinic acid, and fluorouracil versus folinic acid and fluorouracil alone for gemcitabine-refractory pancreatic cancer: Outcomes from the CONKO-003 trial. J Clin Oncol 32:2423-2429, 2014

14. Rougier $P$, Riess $H$, Manges $R$, et al: Randomised, placebo-controlled, double-blind, parallel-group phase III study evaluating aflibercept in patients receiving first-line treatment with gemcitabine for metastatic pancreatic cancer. Eur J Cancer 49: 2633-2642, 2013
15. Gonçalves A, Gilabert M, François E, et al: BAYPAN study: A double-blind phase III randomized trial comparing gemcitabine plus sorafenib and gemcitabine plus placebo in patients with advanced pancreatic cancer. Ann Oncol 23:2799-2805, 2012

16. Kindler $\mathrm{HL}$, loka T, Richel DJ, et al: Axitinib plus gemcitabine versus placebo plus gemcitabine in patients with advanced pancreatic adenocarcinoma: A double-blind randomised phase 3 study. Lancet Oncol 12:256-262, 2011

17. da Cunha Santos G, Dhani N, Tu D, et al: Molecular predictors of outcome in a phase 3 study of gemcitabine and erlotinib therapy in patients with advanced pancreatic cancer: National Cancer Institute of Canada Clinical Trials Group Study PA.3. Cancer 116:5599-5607, 2010

18. Moinpour CM, Vaught NL, Goldman B, et al: Pain and emotional well-being outcomes in Southwest Oncology Group-directed intergroup trial S0205: a phase III study comparing gemcitabine plus cetuximab versus gemcitabine as first-line therapy in patients with advanced pancreas cancer. J Clin Oncol 28: 3611-3616, 2010

19. Colucci G, Labianca R, Di Costanzo F, et al: Randomized phase III trial of gemcitabine plus cisplatin compared with single-agent gemcitabine as first-line treatment of patients with advanced pancreatic cancer: The GIP-1 study. J Clin Oncol 28: 1645-1651, 2010 
20. Dahan $L$, Bonnetain F, Ychou $M$, et al: Combination 5-fluorouracil, folinic acid and cisplatin (LV5FU2CDDP) followed by gemcitabine or the reverse sequence in metastatic pancreatic cancer: Final results of a randomised strategic phase III trial (FFCD 0301). Gut 59 : 1527-1534, 2010

21. Cunningham $D$, Chau $I$, Stocken DD, et al: Phase III randomized comparison of gemcitabine versus gemcitabine plus capecitabine in patients with advanced pancreatic cancer. J Clin Oncol 27 5513-5518, 2009

22. Van Cutsem E, Vervenne WL, Bennouna J, et al: Phase III trial of bevacizumab in combination with gemcitabine and erlotinib in patients with metastatic pancreatic cancer. J Clin Oncol 27:2231-2237, 2009

23. Poplin E, Feng $Y$, Berlin J, et al: Phase III randomized study of gemcitabine and oxaliplatin versus gemcitabine (fixed-dose rate infusion) compared with gemcitabine (30-minute infusion) in patients with pancreatic carcinoma E6201: A trial of the Eastern Cooperative Oncology Group. J Clin Oncol 27:3778-3785, 2009

24. Bernhard J, Dietrich D, Scheithauer W, et al: Clinical benefit and quality of life in patients with advanced pancreatic cancer receiving gemcitabine plus capecitabine versus gemcitabine alone: A randomized multicenter phase III clinical trial_-SAKK 44/00-CECOG/PAN.1.3.001. J Clin Oncol 26:3695-3701, 2008

25. Herrmann R, Bodoky G, Ruhstaller $T$, et al: Gemcitabine plus capecitabine compared with gemcitabine alone in advanced pancreatic cancer: A randomized, multicenter, phase III trial of the Swiss Group for Clinical Cancer Research and the Central European Cooperative Oncology Group. J Clin Oncol 25:2212-2217, 2007

26. Moore MJ, Goldstein D, Hamm J, et al: Erlotinib plus gemcitabine compared with gemcitabine alone in patients with advanced pancreatic cancer: A phase III trial of the National Cancer Institute of Canada Clinical Trials Group. J Clin Oncol 25: 1960-1966, 2007

27. Abou-Alfa GK, Letourneau R, Harker G, et al: Randomized phase III study of exatecan and gemcitabine compared with gemcitabine alone in untreated advanced pancreatic cancer. J Clin Oncol 24 4441-4447, 2006

28. Stathopoulos GP, Syrigos K, Aravantinos G, et al: A multicenter phase III trial comparing irinotecangemcitabine (IG) with gemcitabine $(G)$ monotherapy as first-line treatment in patients with locally advanced or metastatic pancreatic cancer. Br J Cancer 95:587-592, 2006

29. Heinemann V, Quietzsch D, Gieseler F, et al: Randomized phase III trial of gemcitabine plus cisplatin compared with gemcitabine alone in advanced pancreatic cancer. J Clin Oncol 24:3946-3952, 2006

30. Reni M, Cordio S, Milandri $C$, et al: Gemcitabine versus cisplatin, epirubicin, fluorouracil, and gemcitabine in advanced pancreatic cancer: A randomised controlled multicentre phase III trial. Lancet Oncol 6 : 369-376, 2005

31. Louvet $C$, Labianca $R$, Hammel $P$, et al: Gemcitabine in combination with oxaliplatin compared with gemcitabine alone in locally advanced or metastatic pancreatic cancer: Results of a GERCOR and GISCAD phase III trial. J Clin Oncol 23: 3509-3516, 2005

32. Rocha Lima CM, Green MR, Rotche R, et al: Irinotecan plus gemcitabine results in no survival advantage compared with gemcitabine monotherapy in patients with locally advanced or metastatic pancreatic cancer despite increased tumor response rate. J Clin Oncol 22:3776-3783, 2004

33. Berlin JD, Catalano $P$, Thomas JP, et al: Phase III study of gemcitabine in combination with fluorouracil versus gemcitabine alone in patients with advanced pancreatic carcinoma: Eastern Cooperative Oncology Group Trial E2297. J Clin Oncol 20: 3270-3275, 2002

34. Maisey $N$, Chau I, Cunningham D, et al: Multicenter randomized phase III trial comparing protracted venous infusion (PVI) fluorouracil (5-FU) with PVI 5-FU plus mitomycin in inoperable pancreatic cancer. J Clin Oncol 20:3130-3136, 2002

35. Goldstein D, El-Maraghi $\mathrm{RH}$, Hammel $P$, et al: nab-Paclitaxel plus gemcitabine for metastatic pancreatic cancer: Long-term survival from a phase III trial. J Natl Cancer Inst 107:107, 2015

36. Pawlik TM, Laheru D, Hruban $\mathrm{RH}$, et al: Evaluating the impact of a single-day multidisciplinary clinic on the management of pancreatic cancer. Ann Surg Oncol 15:2081-2088, 2008

37. Hoos WA, James PM, Rahib L, et al: Pancreatic cancer clinical trials and accrual in the United States. J Clin Oncol 31:3432-3438, 2013

38. Ma C, Bandukwala $S$, Burman $D$, et al: Interconversion of three measures of performance status: An empirical analysis. Eur J Cancer 46:3175-3183, 2010

39. Mahaseth $H$, Brutcher $E$, Kauh J, et al: Modified FOLFIRINOX regimen with improved safety and maintained efficacy in pancreatic adenocarcinoma. Pancreas 42:1311-1315, 2013

40. Wang-Gillam A, Li CP, Bodoky G, et al: Nanoliposomal irinotecan with fluorouracil and folinic acid in metastatic pancreatic cancer after previous gemcitabine-based therapy (NAPOLI-1): A global, randomised, open-label, phase 3 trial. Lancet 387 545-557, 2016

41. Burris HA III, Moore MJ, Andersen J, et al: Improvements in survival and clinical benefit with gemcitabine as first-line therapy for patients with advanced pancreas cancer: A randomized trial. J Clin Oncol 15:2403-2413, 1997

42. Rahma OE, Duffy $A$, Liewehr DJ, et al: Second-line treatment in advanced pancreatic can cer: A comprehensive analysis of published clinical trials. Ann Oncol 24:1972-1979, 2013

43. Morrison RS, Augustin R, Souvanna $P$, et al: America's care of serious illness: a state-by-state report card on access to palliative care in our nation's hospitals. J Palliat Med 14:1094-1096, 2011

44. Kelley AS, Morrison RS: Palliative care for the seriously ill. N Engl J Med 373:747-755, 2015

45. Seicean A: Celiac plexus neurolysis in pancreatic cancer: The endoscopic ultrasound approach. World J Gastroenterol 20:110-117, 2014

46. Wong GY, Schroeder DR, Carns PE, et al: Effect of neurolytic celiac plexus block on pain relief, quality of life, and survival in patients with unresectable pancreatic cancer: A randomized controlled trial. JAMA 291:1092-1099, 2004

47. Amr YM, Makharita MY: Neurolytic sympathectomy in the management of cancer pain-time effect: A prospective, randomized multicenter study. J Pain Symptom Manage 48:944-956, 2014

48. Damerla V, Gotlieb V, Larson H, et al: Pancreatic enzyme supplementation in pancreatic cancer. J Support Oncol 6:393-396, 2008
49. Bruno MJ, Haverkort EB, Tijssen GP, et al: Placebo controlled trial of enteric coated pancreatin microsphere treatment in patients with unresectable cancer of the pancreatic head region. Gut 42:92-96, 1998

50. Boulay BR, Parepally M: Managing malignant biliary obstruction in pancreas cancer: Choosing the appropriate strategy. World J Gastroenterol 20: 9345-9353, 2014

51. Baron TH: Expandable metal stents for the treatment of cancerous obstruction of the gastrointestinal tract. N Engl J Med 344:1681-1687, 2001

52. Maire $F$, Hammel $P$, Ponsot $P$, et al: Long-term outcome of biliary and duodenal stents in palliative treatment of patients with unresectable adenocarcinoma of the head of pancreas. Am J Gastroenterol 101:735-742, 2006

53. Khorana AA, Ahrendt $S A$, Ryan $C K$, et al: Tissue factor expression, angiogenesis, and thrombosis in pancreatic cancer. Clin Cancer Res 13: 2870-2875, 2007

54. Khorana AA: Venous thromboembolism and prognosis in cancer. Thromb Res 125:490-493, 2010

55. Khorana AA, Francis CW, Culakova $E$, et al: Thromboembolism is a leading cause of death in cancer patients receiving outpatient chemotherapy. J Thromb Haemost 5:632-634, 2007

56. Lyman $\mathrm{GH}$, Bohlke $K$, Khorana $A A$, et al: Venous thromboembolism prophylaxis and treatment in patients with cancer: American Society of Clinical Oncology clinical practice guideline update 2014. J Clin Oncol 33:654-656, 2015

57. Maraveyas A, Waters J, Roy R, et al: Gemcitabine versus gemcitabine plus dalteparin thromboprophylaxis in pancreatic cancer. Eur $\mathrm{J}$ Cancer 48: 1283-1292, 2012

58. Vadhan-Raj S, Zhou X, Benjamin RS, et al: Incidence and risk factors predictive of recurrent venous thromboembolism in patients with cancer at a comprehensive cancer center. J Clin Oncol 29, 2011 (suppl, abstr 9059)

59. Epstein RMSR, Jr. Patient-Centered Communication in Cancer Care: Promoting Healing and Reducing Suffering. Bethesda, MD, National Cancer Institute, 2007

60. Mead H, Cartwright-Smith $L$, Jones $K$, et al: Racial and Ethnic Disparities in U.S. Health Care: A Chartbook. New York, NY, Commonwealth Fund, 2008

61. Lee S, Reha JL, Tzeng CW, et al: Race does not impact pancreatic cancer treatment and survival in an equal access federal health care system. Ann Surg Oncol 20:4073-4079, 2013

62. Dias-Santos D, Ferrone $\mathrm{CR}$, Zheng $\mathrm{H}$, et al: The Charlson age comorbidity index predicts early mortality after surgery for pancreatic cancer. Surgery 157 : 881-887, 2015

63. Hurria A, Togawa K, Mohile SG, et al: Predicting chemotherapy toxicity in older adults with cancer: A prospective multicenter study. J Clin Oncol 29:3457-3465, 2011

64. Attard CL, Brown S, Alloul K, et al: Costeffectiveness of FOLFIRINOX for first-line treatment of metastatic pancreatic cancer. Curr Oncol 21:e41-e51, 2014

65. Chiorean EG, Whiting S, Binder G, et al: Costeffectiveness of nab-paclitaxel plus gemcitabine versus erlotinib plus gemcitabine in metastatic pancreatic cancer. J Clin Oncol 32, 2014 (suppl, abstr 353)

66. Abbruzzese JL, Hess KR: New option for the initial management of metastatic pancreatic cancer? J Clin Oncol 32:2405-2407, 2014 


\section{AUTHORS' DISCLOSURES OF POTENTIAL CONFLICTS OF INTEREST}

Metastatic Pancreatic Cancer: American Society of Clinical Oncology Clinical Practice Guideline

The following represents disclosure information provided by authors of this manuscript. All relationships are considered compensated. Relationships are self-held unless noted. I = Immediate Family Member, Inst = My Institution. Relationships may not relate to the subject matter of this manuscript. For more information about ASCO's conflict of interest policy, please refer to www.asco.org/rwc or jco.ascopubs.org/site/ifc.

Davendra P.S. Sohal

Research Funding: Novartis (Inst), Celgene (Inst), OncoMed (Inst), Bayer (Inst)

Pamela B. Mangu

No relationship to disclosure

\section{Alok A. Khorana}

Consulting or Advisory Role: Eli Lilly

Research Funding: Eli Lilly (Inst), ImClone Systems (Inst), Gilead Sciences (Inst), Merck (Inst), Berg (Inst)

\section{Manish A. Shah}

Honoraria: Sanofi, LEO Pharma, AngioDynamics, Genentech, Daiichi Sankyo, Janssen Pharmaceuticals (a Johnson \& Johnson co.), Boehringer Ingelheim, Halozyme Therapeutics, Pfizer

Consulting or Advisory Role: Sanofi, LEO Pharma, AngioDynamics, Daiichi Sankyo, Genentech, Janssen Pharmaceuticals (a Johnson \& Johnson co.), Boehringer Ingelheim, Halozyme Therapeutics

Research Funding: LEO Pharma, Amgen (Inst)

Travel, Accommodations, Expenses: Janssen Pharmaceuticals (a Johnson \& Johnson co.), AngioDynamics, Halozyme Therapeutics

\section{Philip A. Philip}

Honoraria: Celgene, Amgen, Roche, Sanofi, Bayer, Bristol-Myers Squibb, Novartis, Ipsen, Halozyme Therapeutics, Merrimack Pharmaceuticals Consulting or Advisory Role: Celgene, Novartis, Halozyme Therapeutics, Merrimack Pharmaceuticals, Gilead Sciences, Ipsen

Speakers' Bureau: Celgene, Bayer, Amgen, Roche, Sanofi

Research Funding: Bayer (Inst), Incyte (Inst), Karyopharm Therapeutics (Inst), Merck (Inst), Taiho Pharmaceutical (Inst), Momenta

Pharmaceuticals (Inst), Novartis (Inst), Plexxikon (Inst), Immunomedics (Inst), Regeneron Pharmaceuticals (Inst), Genentech (Inst)

Travel, Accommodations, Expenses: Celgene

\section{Eileen M. O'Reilly}

Consulting or Advisory Role: Celgene, Hexal (I), Aduro Biotech, Astellas Pharma (I), Celsion (I), Cipla, Eli Lilly (I), Exelixis (I), IntegraGen Genomics (I), Jennerex Biotherapeutics (I), MedImmune, Novartis (I), Pharmacyclics, Sanofi, Silenseed, Vicus Therapeutics, Gilead Sciences, Merrimack Pharmaceuticals

Research Funding: Momenta Pharmaceuticals (Inst), Incyte (Inst), Immunomedics (Inst), Myriad Genetics (Inst), OncoMed Pharmaceuticals (Inst), Clovis Oncology (Inst), Sanofi (Inst), Celgene (Inst), Polaris Pharmaceuticals (Inst), Bristol-Myers Squibb (Inst), AstraZeneca (Inst), MedImmune (Inst)

\section{Hope E. Uronis}

Employment: GeneCentric Diagnostics (I)

Research Funding: Merrimack Pharmaceuticals, Genentech, BristolMyers Squibb, MacroGenics, Advaxis, Threshold Pharmaceuticals

\section{Ramesh K. Ramanathan}

Honoraria: Celgene, Eli Lilly, Cerulean Pharma, Pharmacyclics Consulting or Advisory Role: Celgene, Eli Lilly

Research Funding: AbbVie, Genetech, Bayer, Halozyme Therapeutics, Vaccinex, Celgene, Tekmira, Schering-Plough, BioMarin, Merrimack Pharmaceuticals, Verastem

\section{Christopher H. Crane}

Honoraria: Vertex Pharmaceuticals, EMD Serono

Consulting or Advisory Role: Vertex Pharmaceuticals, EMD Serono

\section{Anitra Engebretson}

Honoraria: AbbVie

Travel, Accommodations, Expenses: AbbVie

\section{Joseph T. Ruggiero}

No relationship to disclose

\section{Mehmet S. Copur}

Honoraria: Amgen

Michelle Lau

Travel, Accommodations, Expenses: Agendia NV

\section{Susan Urba}

Honoraria: Eisai, Merck

Consulting or Advisory Role: Eisai, Merck

Travel, Accommodations, Expenses: Eisai, Merck

Dan Laheru

No relationship to disclose 


\section{Acknowledgment}

We thank E. Gabriela Chiorean, Christopher Willett, Neelima Denduluri, Abhishek Tripathi, and the Clinical Practice Guidelines Committee for thoughtful reviews and insightful comments on this guideline document. We also acknowledge the significant contributions to the systematic review and development of the manuscript by Shannon E. McKernin and Brittany E. Harvey. Dedicated to T.B.

\section{Appendix}

\begin{tabular}{|c|c|}
\hline Name (and Designation) & Affiliation/Institution \\
\hline Davendra P.S. Sohal, MD, MPH (co-chair) & Cleveland Clinic, Cleveland, $\mathrm{OH}$ \\
\hline Daniel Laheru, MD (co-chair) & Sidney Kimmel Comprehensive Cancer Center, Baltimore, MD \\
\hline Alok A. Khorana, MD & Cleveland Clinic, Cleveland, $\mathrm{OH}$ \\
\hline Manish A. Shah, MD & The Weill Cornell Medical Center, New York, NY \\
\hline Eileen M. O’Reilly, MD & Memorial Sloan Kettering Cancer Center, New York, NY \\
\hline Hope E. Uronis, MD & Duke University, Durham, NC \\
\hline Ramesh K. Ramanathan, MD & Mayo Clinic, Scottsdale, AZ \\
\hline Christopher H. Crane, MD & The University of Texas MD Anderson Cancer Center, Houston, TX \\
\hline Philip A. Philip, MD, PhD & Karmanos Cancer Institute, Detroit, MI \\
\hline Anitra Engebretson (patient representative) & Portland, OR \\
\hline Joseph T. Ruggiero, MD & Weill Cornell Medical College, New York, NY \\
\hline Mehmet S. Copur, MD (PGIN representative) & St Francis Medical Center, Grand Island, NE \\
\hline Michelle Lau (PGIN representative) & Private practice, Tempe, AZ \\
\hline Susan Urba, MD & University of Michigan Cancer Center, Ann Arbor, MI \\
\hline
\end{tabular}

